\title{
Stability of Impulsive Cohen-Grossberg Neural Networks with Time-Varying Delays and Reaction-Diffusion Terms
}

\author{
Jinhua Huang, ${ }^{1}$ Jiqing Liu, ${ }^{1}$ and Guopeng Zhou ${ }^{2}$ \\ ${ }^{1}$ College of Electric and Electronic Engineering, Wuhan Institute of Shipbuilding Technology, Wuhan, Hubei 430050, China \\ ${ }^{2}$ College of Electronic and Information Engineering, Hubei University of Science and Technology, Xianning, Hubei 437100, China \\ Correspondence should be addressed to Guopeng Zhou; zhgpeng@163.com
}

Received 29 December 2012; Accepted 23 February 2013

Academic Editor: Qi Luo

Copyright (c) 2013 Jinhua Huang et al. This is an open access article distributed under the Creative Commons Attribution License, which permits unrestricted use, distribution, and reproduction in any medium, provided the original work is properly cited.

\begin{abstract}
This work concerns the stability of impulsive Cohen-Grossberg neural networks with time-varying delays and reaction-diffusion terms as well as Dirichlet boundary condition. By means of Poincaré inequality and Gronwall-Bellman-type impulsive integral inequality, we summarize some new and concise sufficient conditions ensuring the global exponential stability of equilibrium point. The proposed criteria are relevant to the diffusion coefficients and the smallest positive eigenvalue of corresponding Dirichlet Laplacian. In conclusion, two examples are illustrated to demonstrate the effectiveness of our obtained results.
\end{abstract}

\section{Introduction}

Cohen-Grossberg neural networks (CGNNs) were introduced by Cohen and Grossberg in 1983 [1] and have been a hot topic due to their important applications in various fields such as parallel computation, associative memory, image processing, and optimization problems.

By reason that time delays are unavoidably encountered for the finite switching speed of neurons and amplifiers in the implementation of neural networks, a more powerful model of delayed Cohen-Grossberg neural networks (DCGNNs) is afterwards proposed. This kind of mathematical models is widely applied in dynamic image processing and pattern recognition problems. It is worth noting that all these applications depend heavily on the dynamical behaviors such as stability, convergence, and oscillatory [2-6]. Meanwhile, stability is an important consideration in the designs and applications of neural networks. The stability of delayed neural networks is a subject of current interest, and therefore considerable theoretical efforts have been put into this topic followed by a large number of stability criteria reported; for example, see [7-12] and the references therein.

In real world, however, many evolutionary processes are characterized by abrupt changes at certain instants which may be caused by switching phenomena, frequency changes, or other sudden noises. As such, in the past few years, scientists have become gradually interested in the influence that impulses may have on the CGNNs and DCGNNs, thus obtaining some related results; for example, see [13-18] and the references therein.

Actually, besides impulsive effects, we have to recognize that diffusion effects are also nonignorable in reality as diffusion is unavoidable when electrons are moving in asymmetric electromagnetic fields. On this account, the model of neural networks with both impulses and diffusion should be more effective for describing the evolutionary process of practical systems. Based on this consideration, we wonder what the influence of diffusion on the stability of CGNNs and DCGNNs is.

So far there have appeared a few theoretical achievements [19-29] on the stability of impulsive reaction-diffusion neural networks with or without delays. Particularly, in [21-26], the main research technique is the impulsive differential inequality whereby the authors discussed the stability of equilibrium point and provided a series of sufficient conditions independent of diffusion. From these results, we fail to see the influence of diffusion on the stability of CGNNs and DCGNNs.

Encouragingly, recently there were reported some new results on the stability of CGNNs and DCGNNs in $[19,20$, 27]; thereinto, the presented stability criteria derived from 
the impulsive differential inequality are related to the diffusion terms, and thereby we know the diffusion do contribute to the stability of impulsive neural networks.

In this paper, different from $[20,27]$, we shall consider the case where the boundary condition is Dirichlet boundary condition rather than Neumann boundary condition. Moreover, unlike [19], we shall utilize the new method of Poincarè inequality to deal with the reaction-diffusion terms, and Gronwall-Bellman-type impulsive integral inequality is also introduced for stability analysis. The obtained results show that not only the reaction-diffusion coefficients but also the first eigenvalue of corresponding Dirichlet Laplacian can affect the stability.

The rest of this paper is structured as follows. In Section 2, the model of impulsive delayed Cohen-Grossberg neural networks with reaction-diffusion terms as well as Dirichlet boundary condition is outlined and some facts and lemmas are introduced for later reference. By the new agencies of Gronwall-Bellman-type impulsive integral inequality and Poincaré inequality, we discuss the global exponential stability of equilibrium point and develop some new and concise algebraic criteria in Section 3. To conclude, two illustrative examples are given in Section 4 to verify the effectiveness of our results.

\section{Preliminaries}

Let $R^{n}$ denote the $n$-dimensional Euclidean space, and let $\Omega \subset$ $R^{m}$ be an open bounded domain with smooth boundary $\partial \Omega$ and mes $\Omega>0$. Let $R_{+}=[0, \infty)$ and $t_{0} \in R_{+}$.

Consider the following impulsive CGNN with timevarying delays and reaction-diffusion terms:

$$
\begin{gathered}
\frac{\partial u_{i}(t, x)}{\partial t}=\sum_{s=1}^{m} \frac{\partial}{\partial x_{s}}\left(D_{i s} \frac{\partial u_{i}(t, x)}{\partial x_{s}}\right) \\
-a_{i}\left(u_{i}(t, x)\right)\left[\omega_{i}\left(u_{i}(t, x)\right)-\sum_{j=1}^{n} b_{i j} f_{j}\left(u_{j}(t, x)\right)\right. \\
\left.\quad-\sum_{j=1}^{n} c_{i j} f_{j}\left(u_{j}\left(t-\tau_{j}(t), x\right)\right)\right], \\
t \geq t_{0}, t \neq t_{k}, x \in \Omega, i=1,2, \ldots, n, k=1,2, \ldots, \\
u_{i}\left(t_{k}+0, x\right)=u_{i}\left(t_{k}, x\right)+P_{i k}\left(u_{i}\left(t_{k}, x\right)\right), \\
x \in \Omega, k=1,2, \ldots, \quad i=1,2, \ldots, n,
\end{gathered}
$$

where $n$ corresponds to the numbers of units in a neural network, $x=\left(x_{1}, \ldots, x_{m}\right)^{T} \in \Omega, u_{i}(t, x)$ denotes the state of the $i$ th neuron at time $t$ and in space $x, D_{i s}=$ const $>0$ represents transmission diffusion of the $i$ th unit, $a_{i}\left(u_{i}(t, x)\right)$ represents the amplification function, $\omega_{i}\left(u_{i}(t, x)\right)$ is the appropriate behavior function, activation function $f_{j}\left(u_{j}(t, x)\right)$ stands for the output of the $j$ th unit at time $i$ and in space $x$ and $b_{i j}$ and $c_{i j}$ are constants: $b_{i j}$ indicates the connection strength of the $j$ th unit on the $i$ th unit at time $t$ and in space $x$, while $c_{i j}$ denotes the connection weight of the $j$ th unit on the $i$ th unit at time $t-\tau_{j}(t)$ and in space $x$, where $\tau_{j}(t)$ corresponds to the transmission delay along the axon of the jth unit satisfying $0 \leq \tau_{j}(t) \leq \tau$ ( $\tau=$ const) and $\dot{\tau}_{j}(t)<1-(1 / h)(h>0) .\left\{t_{k}\right\}(k=1,2, \ldots)$ is the sequence of impulsive moments meeting $0 \leq t_{0}<t_{1}<t_{2}<\cdots$ and $\lim _{k \rightarrow \infty} t_{k}=\infty ; u_{i}\left(t_{k}+0, x\right)$ and $u_{i}\left(t_{k}-0, x\right)$ represent the right-hand and left-hand limit of $u_{i}(t, x)$ at time $t_{k}$ and in space $x$, respectively. $P_{i k}\left(u_{i}\left(t_{k}, x\right)\right)$ stands for the abrupt change of $u_{i}(t, x)$ at impulsive moment $t_{k}$ and in space $x$.

Denote by $u(t, x)=u\left(t, x ; t_{0}, \varphi\right), u \in R^{n}$, the solution of systems (1)-(2), satisfying the initial condition

$$
u\left(s, x ; t_{0}, \varphi\right)=\varphi(s, x), \quad t_{0}-\tau \leq s \leq t_{0}, x \in \Omega,
$$

and Dirichlet boundary condition

$$
u\left(t, x ; t_{0}, \varphi\right)=0, \quad t \geq t_{0}, x \in \partial \Omega,
$$

where the vector-valued function $\varphi(s, x)=\left(\varphi_{1}(s, x), \ldots\right.$, $\left.\varphi_{n}(s, x)\right)^{T}$ is such that $\int_{\Omega} \sum_{i=1}^{n} \varphi_{i}^{2}(s, x) d x$ is bounded on $\left[t_{0}-\right.$ $\left.\tau, t_{0}\right]$.

The solution $u(t, x)=u\left(t, x ; t_{0}, \varphi\right)=\left(u_{1}\left(t, x ; t_{0}, \varphi\right), \ldots\right.$, $\left.u_{n}\left(t, x ; t_{0}, \varphi\right)\right)^{T}$ of problems (1)-(4) is, for the time variable $t$, a piecewise continuous function with the first kind discontinuity at the points $t_{k}(k=1,2, \ldots)$, where it is left-continuous; that is, the following relations are valid:

$$
\begin{gathered}
u_{i}\left(t_{k}-0, x\right)=u_{i}\left(t_{k}, x\right), \\
u_{i}\left(t_{k}+0, x\right)=u_{i}\left(t_{k}, x\right)+P_{i k}\left(u_{i}\left(t_{k}, x\right)\right) .
\end{gathered}
$$

Throughout this paper, we define the norm of $u\left(t, x ; t_{0}, \varphi\right)$ as

$$
\left\|u\left(t, x ; t_{0}, \varphi\right)\right\|_{\Omega}=\sqrt{\sum_{i=1}^{n} \int_{\Omega} u_{i}^{2}\left(t, x ; t_{0}, \varphi\right) d x}
$$

and make the following assumptions for convenience.

(H1) $a_{i}(\cdot): R \rightarrow R^{+}$is continuous and bounded; that is, there exist constants $\underline{a}_{i}$ and $\bar{a}_{i}$ such that

$$
0<\underline{a}_{i} \leq a_{i}(\zeta) \leq \bar{a}_{i}<\infty, \quad \text { for } i=1, \ldots, n .
$$

$(\mathrm{H} 2) \omega_{i}(\cdot): R \rightarrow R$ is continuous and $\omega_{i}(0)=0$; moreover, there exists constant $p_{i}>0$ such that

$$
\frac{\omega_{i}\left(\zeta_{1}\right)-\omega_{i}\left(\zeta_{2}\right)}{\zeta_{1}-\zeta_{2}} \geq p_{i}>0, \quad \text { for } \zeta_{1} \neq \zeta_{2}, i=1, \ldots, n
$$

(H3) $f_{i}(\cdot): R \rightarrow R$ is continuous and $f_{i}(0)=0$; furthermore, there exists constant $l_{i}>0$ such that

$$
l_{i}=\sup _{\zeta_{1} \neq \zeta_{2}} \frac{f_{i}\left(\zeta_{1}\right)-f_{i}\left(\zeta_{2}\right)}{\zeta_{1}-\zeta_{2}} \text { for } \zeta_{1} \neq \zeta_{2}, \quad i=1,2, \ldots, n \text {. }
$$

(H4) $P_{i k}(\cdot): R \rightarrow R$ is continuous and $P_{i k}(0)=0$ for $i=$ $1,2, \ldots, n$ and $k=1,2, \ldots$ 
In the light of $(\mathrm{H} 1)-(\mathrm{H} 4)$, it is easy to see that problems (1)-(2) admit an equilibrium point $u=0$.

Definition 1. The equilibrium point $u=0$ of problems (1)(2) is said to be globally exponentially stable if there exist constants $\kappa>0$ and $M \geq 1$ such that

$$
\left\|u\left(t, x ; t_{0}, \varphi\right)\right\|_{\Omega} \leq M \overline{\|\varphi\|_{\Omega}} e^{-\kappa\left(t-t_{0}\right)}, \quad t \geq t_{0},
$$

where ${\overline{\|\varphi\|_{\Omega}^{2}}}_{\Omega}^{2} \sup _{t_{0}-\tau \leq s \leq t_{0}} \sum_{i=1}^{n} \int_{\Omega} \varphi_{i}^{2}(s, x) d x$.

Lemma 2 (see [30] (Gronwall-Bellman-type impulsive integral inequality)). Assume the following.

(A1) The sequence $\left\{t_{k}\right\}$ satisfies $0 \leq t_{0}<t_{1}<t_{2}<\cdots$, with $\lim _{k \rightarrow \infty} t_{k}=\infty$.

(A2) $q \in P C^{1}\left[R_{+}, R\right]$ and $q(t)$ is left-continuous at $t_{k}, k=$ $1,2, \ldots$.

(A3) $p \in C\left[R_{+}, R_{+}\right]$and for $k=1,2, \ldots$,

$$
q(t) \leq c+\int_{t_{0}}^{t} p(s) q(s) d s+\sum_{t_{0}<t_{k}<t} \eta_{k} q\left(t_{k}\right), \quad t \geq t_{0},
$$

where $\eta_{k} \geq 0$ and $c=$ const. Then,

$$
q(t) \leq c \prod_{t_{0}<t_{k}<t}\left(1+\eta_{k}\right) \exp \left(\int_{t_{0}}^{t} p(s) d s\right), \quad t \geq t_{0} .
$$

Lemma 3 (see [31] (Poincaré inequality)). Let $\mathcal{S}$ be a bounded region in $R^{n}, v(x) \in C^{1}(\mathcal{S})$, and $v=0$ on the boundary of $\mathcal{S}$; then

$$
\lambda_{1} \int_{\mathcal{S}} v^{2}(x) d x \leq \int_{\mathcal{S}}|\nabla v(x)|^{2} d x
$$

where $\lambda_{1}$ is the smallest positive eigenvalue of the following problem:

$$
\Delta \Psi(x)+\lambda \Psi(x)=0, \quad x \in \mathcal{S}, \quad \Psi(x)=0, \quad x \in \partial \mathcal{S} .
$$

Lemma 4. If $a>0$ and $b>0$, then $a b \leq(1 / \varepsilon) a^{2}+\varepsilon b^{2}$ holds for any $\varepsilon>0$.

\section{Main Results}

Theorem 5. Assume the following.

(1) $\underline{D}=\min \left\{D_{i s}: i=1, \ldots, n ; s=1, \ldots, m\right\}>0$ and denote $2 \underline{D} \lambda_{1}=\chi$.

(2) $P_{i k}\left(u_{i}\left(t_{k}, x\right)\right)=-\theta_{i k} u_{i}\left(t_{k}, x\right), 0 \leq \theta_{i k} \leq 2$.

(3) There exists a constant $\gamma>0$ satisfying $\gamma+\lambda+h \rho e^{\gamma \tau}>$ 0 and $\lambda+h \rho e^{\gamma \tau}<0$, where

$$
\begin{gathered}
\lambda=\max _{i=1, \ldots, n}\left(-\chi-2 \underline{a}_{i} p_{i}+\bar{a}_{i} \sum_{j=1}^{n} b_{i j}^{2}+\bar{a}_{i} \sum_{j=1}^{n} c_{i j}^{2}\right)+\rho, \\
\rho=\max _{i=1, \ldots, n}\left(l_{i}^{2}\right) \sum_{i=1}^{n} \bar{a}_{i} .
\end{gathered}
$$

Then, the equilibrium point $u=0$ of systems (1)-(2) is globally exponentially stable with convergence rate $-\left(\lambda+h \rho e^{\gamma \tau}\right) / 2$.

Proof. Multiplying both sides of (1) by $u_{i}(t, x)$, we get

$$
\begin{array}{r}
\frac{\partial u_{i}^{2}(t, x)}{\partial t}=2 \sum_{s=1}^{m} u_{i}(t, x) \frac{\partial}{\partial x_{s}}\left(D_{i s} \frac{\partial u_{i}(t, x)}{\partial x_{s}}\right) \\
-2 u_{i}(t, x) a_{i}\left(u_{i}(t, x)\right) \\
\times\left[\begin{array}{l}
\omega_{i}\left(u_{i}(t, x)\right)-\sum_{j=1}^{n} b_{i j} f_{j}\left(u_{j}(t, x)\right) \\
\left.-\sum_{j=1}^{n} c_{i j} f_{j}\left(u_{j}\left(t-\tau_{j}(t), x\right)\right)\right], \\
t \geq t_{0}, t \neq t_{k}, x \in \Omega, k=1,2, \ldots,
\end{array}\right.
\end{array}
$$

which yields, after integrating with respect to spatial variable $x$ on $\Omega$,

$$
\begin{gathered}
\frac{d\left(\int_{\Omega} u_{i}^{2}(t, x) d x\right)}{d t}=J_{1}+J_{2}, \\
t \geq t_{0}, \quad t \neq t_{k}, \quad k=1,2, \ldots,
\end{gathered}
$$

where $J_{1}=2 \int_{\Omega} \sum_{s=1}^{m}\left(u_{i}(t, x)\left(\partial / \partial x_{s}\right)\left(D_{i s}\left(\partial u_{i}(t, x)\right) / \partial x_{s}\right)\right) d x$,

$$
\begin{aligned}
J_{2}= & -2 \int_{\Omega} u_{i}(t, x) a_{i}\left(u_{i}(t, x)\right) \\
\times & {\left[\omega_{i}\left(u_{i}(t, x)\right)-\sum_{j=1}^{n} b_{i j} f_{j}\left(u_{j}(t, x)\right)\right.} \\
& \left.-\sum_{j=1}^{n} c_{i j} f_{j}\left(u_{j}\left(t-\tau_{j}(t), x\right)\right)\right] d x .
\end{aligned}
$$

By combining Green formula, Dirichlet boundary condition, Lemma 3, and condition (1) of Theorem 5, we obtain

$$
\begin{aligned}
J_{1} & =-2 \sum_{s=1}^{m} \int_{\Omega} D_{i s}\left(\frac{\partial u_{i}(t, x)}{\partial x_{s}}\right)^{2} d x \\
& \leq-2 \underline{D} \lambda_{1} \int_{\Omega} u_{i}^{2}(t, x) d x \triangleq-\chi \int_{\Omega} u_{i}^{2}(t, x) d x .
\end{aligned}
$$


Moreover, it follows from assumptions (H1), (H2), and (H3) that

$$
\begin{aligned}
& 2 \int_{\Omega} u_{i}(t, x) a_{i}\left(u_{i}(t, x)\right) \omega_{i}\left(u_{i}(t, x)\right) d x \\
& \geq 2 \underline{a}_{i} p_{i} \int_{\Omega}\left|u_{i}(t, x)\right|^{2} d x \\
& 2 \int_{\Omega} u_{i}(t, x) a_{i}\left(u_{i}(t, x)\right) \sum_{j=1}^{n} b_{i j} f_{j}\left(u_{j}(t, x)\right) d x \\
& \leq \bar{a}_{i} \sum_{j=1}^{n} \int_{\Omega}\left(b_{i j}^{2} u_{i}^{2}(t, x)+f_{j}^{2}\left(u_{j}(t, x)\right)\right) d x \\
& \leq \bar{a}_{i} \sum_{j=1}^{n} \int_{\Omega} 2\left|b_{i j}\left\|u_{i}(t, x)\right\| f_{j}\left(u_{j}(t, x)\right)\right| d x \\
& \leq \bar{a}_{i} \sum_{j=1}^{n} \int_{\Omega}\left(b_{i j}^{2} u_{i}^{2}(t, x)+l_{j}^{2} u_{j}^{2}(t, x)\right) d x, \\
& 2 \int_{\Omega} u_{i}(t, x) a_{i}\left(u_{i}(t, x)\right) \sum_{j=1}^{n} c_{i j} f_{j}\left(u_{j}\left(t-\tau_{j}(t), x\right)\right) d x \\
& \leq \bar{a}_{i} \sum_{j=1}^{n} \int_{\Omega}\left(c_{i j}^{2} u_{i}^{2}(t, x)+l_{j}^{2} u_{j}^{2}\left(t-\tau_{j}(t), x\right)\right) d x .
\end{aligned}
$$

Consequently, substituting (19)-(22) into (17) produces

$$
\begin{aligned}
& \frac{d\left(\int_{\Omega} u_{i}^{2}(t, x) d x\right)}{d t} \\
& \leq-\chi \int_{\Omega} u_{i}^{2}(t, x) d x-2 \underline{a}_{i} p_{i} \int_{\Omega} u_{i}^{2}(t, x) d x \\
& \quad+\bar{a}_{i} \sum_{j=1}^{n} \int_{\Omega}\left(b_{i j}^{2} u_{i}^{2}(t, x)+l_{j}^{2} u_{j}^{2}(t, x)\right) d x \\
& \quad+\bar{a}_{i} \sum_{j=1}^{n} \int_{\Omega}\left(c_{i j}^{2} u_{i}^{2}(t, x)+l_{j}^{2} u_{j}^{2}\left(t-\tau_{j}(t), x\right)\right) d x
\end{aligned}
$$

for $t \geq t_{0}, \quad t \neq t_{k}, k=1,2, \ldots$

Now define Lyapunov function $V_{i}(t)$ as $V_{i}(t)=$ $\int_{\Omega} u_{j}^{2}(t, x) d x$. It is not difficult to see that $V_{i}(t)$ is a piecewise continuous function with points of discontinuity of the first kind $t_{k}(k=1,2, \ldots)$, where it is continuous from the left; that is, $V_{i}\left(t_{k}-0\right)=V_{i}\left(t_{k}\right)(k=1,2, \ldots)$. In addition, for $t=t_{k}$ $(k=0,1,2, \ldots)$, we know

$$
V_{i}\left(t_{k}+0\right) \leq V_{i}\left(t_{k}\right), \quad k=0,1,2, \ldots
$$

as $V_{i}\left(t_{0}+0\right) \leq V_{i}\left(t_{0}\right)$ and $u_{i}^{2}\left(t_{k}+0, x\right)=\left(1-\theta_{i k}\right)^{2} u_{i}^{2}\left(t_{k}, x\right) \leq$ $u_{i}^{2}\left(t_{k}, x\right)(k=1,2, \ldots)$, supported by condition 2 of Theorem 5 .
Put $t \in\left(t_{k}, t_{k+1}\right), k=0,1,2, \ldots$ It is derived from (23) that

$$
\begin{aligned}
\frac{d V_{i}(t)}{d t} \leq & \left(-\chi-2 \underline{a}_{i} p_{i}+\bar{a}_{i} \sum_{j=1}^{n} b_{i j}^{2}+\bar{a}_{i} \sum_{j=1}^{n} c_{i j}^{2}\right) V_{i}(t) \\
& +\bar{a}_{i_{i=1, \ldots, n} \max }\left(l_{i}^{2}\right) \sum_{j=1}^{n} V_{j}(t) \\
& +\bar{a}_{i=1, \ldots, n}\left(l_{i}^{2}\right) \sum_{j=1}^{n} V_{j}\left(t-\tau_{j}(t)\right), \\
& t \in\left(t_{k}, t_{k+1}\right), \quad k=0,1,2, \ldots
\end{aligned}
$$

Define function $V(t)$ of the form $V(t)=\sum_{i=1}^{n} V_{i}(t)$ again. From (25), one then reads

$$
\begin{aligned}
\frac{d V(t)}{d t} & \leq \lambda V(t) \\
& +\rho \sum_{j=1}^{n} V_{j}\left(t-\tau_{j}(t)\right), \quad t \in\left(t_{k}, t_{k+1}\right), k=0,1,2, \ldots,
\end{aligned}
$$

where $\rho=\max _{i=1, \ldots, n}\left(l_{i}^{2}\right) \sum_{i=1}^{n} \bar{a}_{i}$ and $\lambda=\max _{i=1, \ldots, n}(-\chi-$ $\left.2 \underline{a}_{i} p_{i}+\bar{a}_{i} \sum_{j=1}^{n} b_{i j}^{2}+\bar{a}_{i} \sum_{j=1}^{n} c_{i j}^{2}\right)+\rho$.

Construct $V^{*}(t)=e^{\gamma\left(t-t_{0}\right)} V(t)$, where $\gamma>0$ satisfies $\gamma+\lambda+h \rho e^{\gamma \tau}>0$ and $\lambda+h \rho e^{\gamma \tau}<0$. Evidently, $V^{*}(t)$ is also a piecewise continuous function with the first kind discontinuous points $t_{k}(k=1,2, \ldots)$, in which it is continuous from the left; that is, $V^{*}\left(t_{k}-0\right)=V^{*}\left(t_{k}\right)(k=1,2, \ldots)$. Moreover, at $t=t_{k}(k=0,1,2, \ldots)$, we find by the use of (24)

$$
V^{*}\left(t_{k}+0\right) \leq V^{*}\left(t_{k}\right), \quad k=0,1,2, \ldots
$$

Set $t \in\left(t_{k}, t_{k+1}\right), k=0,1,2, \ldots$ By virtue of (26), one has

$$
\begin{aligned}
& \frac{d V^{*}(t)}{d t}= \gamma e^{\gamma\left(t-t_{0}\right)} V(t)+e^{\gamma\left(t-t_{0}\right)} \frac{d V(t)}{d t} \\
& \leq \gamma e^{\gamma\left(t-t_{0}\right)} V(t) \\
&+\left(\lambda V(t)+\rho \sum_{j=1}^{n} V_{j}\left(t-\tau_{j}(t)\right)\right) e^{\gamma\left(t-t_{0}\right)} \\
&=(\gamma+\lambda) V^{*}(t)+\rho e^{\gamma\left(t-t_{0}\right)} \sum_{j=1}^{n} V_{j}\left(t-\tau_{j}(t)\right), \\
& t \in\left(t_{k}, t_{k+1}\right), \quad k=0,1,2, \ldots
\end{aligned}
$$

Choose small enough $\varepsilon>0$. Integrating (28) from $t_{k}+\varepsilon$ to $t$ gives

$$
\begin{gathered}
V^{*}(t) \leq V^{*}\left(t_{k}+\varepsilon\right)+(\gamma+\lambda) \int_{t_{k}+\varepsilon}^{t} V^{*}(s) d s \\
+\int_{t_{k}+\varepsilon}^{t} \rho e^{\gamma\left(s-t_{0}\right)} \sum_{j=1}^{n} V_{j}\left(s-\tau_{j}(s)\right) d s \\
t \in\left(t_{k}, t_{k+1}\right), \quad k=0,1,2, \ldots
\end{gathered}
$$


which yields, after letting $\varepsilon \rightarrow 0$ in (29),

$$
\begin{array}{r}
V^{*}(t) \leq V^{*}\left(t_{k}+0\right)+(\gamma+\lambda) \int_{t_{k}}^{t} V^{*}(s) d s \\
+\int_{t_{k}}^{t} \rho e^{\gamma\left(s-t_{0}\right)} \sum_{j=1}^{n} V_{j}\left(s-\tau_{j}(s)\right) d s \\
t \in\left(t_{k}, t_{k+1}\right), k=0,1,2, \ldots
\end{array}
$$

Next, we estimate the value of $V^{*}(t)$ at $t=t_{k+1}, k=0,1$, $2, \ldots$. For small enough $\varepsilon>0$, we put $t=t_{k+1}-\varepsilon$. Now an application of (30) leads to, for $k=0,1,2, \ldots$,

$$
\begin{aligned}
V^{*}\left(t_{k+1}-\varepsilon\right) \leq & V^{*}\left(t_{k}+0\right)+(\gamma+\lambda) \int_{t_{k}}^{t_{k+1}-\varepsilon} V^{*}(s) d s \\
& +\int_{t_{k}}^{t_{k+1}-\varepsilon} \rho e^{\gamma\left(s-t_{0}\right)} \sum_{j=1}^{n} V_{j}\left(s-\tau_{j}(s)\right) d s .
\end{aligned}
$$

If we let $\varepsilon \rightarrow 0$ in (31), there results

$$
\begin{aligned}
& V^{*}\left(t_{k+1}-0\right) \\
& \quad \leq V^{*}\left(t_{k}+0\right)+(\gamma+\lambda) \int_{t_{k}}^{t_{k+1}} V^{*}(s) d s \\
& \quad+\int_{t_{k}}^{t_{k+1}} \rho e^{\gamma\left(s-t_{0}\right)} \sum_{j=1}^{n} V_{j}\left(s-\tau_{j}(s)\right) d s, \quad k=0,1,2, \ldots
\end{aligned}
$$

Note that $V^{*}\left(t_{k+1}-0\right)=V^{*}\left(t_{k+1}\right)$ is applicable for $k=0,1$, $2, \ldots$. Thus,

$$
\begin{aligned}
V^{*}\left(t_{k+1}\right) \leq & V^{*}\left(t_{k}+0\right)+(\gamma+\lambda) \int_{t_{k}}^{t_{k+1}} V^{*}(s) d s \\
& +\int_{t_{k}}^{t_{k+1}} \rho e^{\gamma\left(s-t_{0}\right)} \sum_{j=1}^{n} V_{j}\left(s-\tau_{j}(s)\right) d s
\end{aligned}
$$

holds for $k=0,1,2, \ldots$. By synthesizing (30) and (33), we then arrive at

$$
\begin{array}{r}
V^{*}(t) \leq V^{*}\left(t_{k}+0\right)+(\gamma+\lambda) \int_{t_{k}}^{t} V^{*}(s) d s \\
+\int_{t_{k}}^{t} \rho e^{\gamma\left(s-t_{0}\right)} \sum_{j=1}^{n} V_{j}\left(s-\tau_{j}(s)\right) d s, \\
t \in\left(t_{k}, t_{k+1}\right], k=0,1,2, \ldots
\end{array}
$$

This, together with (27), results in

$$
\begin{aligned}
V^{*}(t) \leq & V^{*}\left(t_{k}\right)+(\gamma+\lambda) \int_{t_{k}}^{t} V^{*}(s) d s \\
& +\int_{t_{k}}^{t} \rho e^{\gamma\left(s-t_{0}\right)} \sum_{j=1}^{n} V_{j}\left(s-\tau_{j}(s)\right) d s
\end{aligned}
$$

for $t \in\left(t_{k}, t_{k+1}\right], k=0,1,2, \ldots$
Recalling the assumptions that $0 \leq \tau_{j}(t) \leq \tau$ and $\dot{\tau}_{j}(t)<$ $1-(1 / h)(h>0)$, we therefore obtain

$$
\begin{aligned}
& \int_{t_{k}}^{t} \rho e^{\gamma\left(s-t_{0}\right)} \sum_{j=1}^{n} V_{j}\left(s-\tau_{j}(s)\right) d s \\
& \quad=\sum_{j=1}^{n} \int_{t_{k}-\tau_{j}\left(t_{k}\right)}^{t-\tau_{j}(t)} \rho e^{\gamma\left(\theta+\tau_{j}(s)-t_{0}\right)} V_{j}(\theta) \frac{1}{1-\dot{\tau}_{j}(s)} d \theta \\
& \quad \leq h \rho e^{\gamma \tau} \sum_{j=1}^{n} \int_{t_{k}-\tau_{j}\left(t_{k}\right)}^{t-\tau_{j}(t)} e^{\gamma\left(\theta-t_{0}\right)} V_{j}(\theta) d \theta
\end{aligned}
$$

Hence,

$$
\begin{aligned}
V^{*}(t) \leq V^{*}\left(t_{k}\right)+(\gamma+\lambda) \int_{t_{k}}^{t} V^{*}(s) d s \\
+h \rho e^{\gamma \tau} \sum_{j=1}^{n} \int_{t_{k}-\tau_{j}\left(t_{k}\right)}^{t-\tau_{j}(t)} e^{\gamma\left(s-t_{0}\right)} V_{j}(s) d s \\
t \in\left(t_{k}, t_{k+1}\right], k=0,1,2, \ldots
\end{aligned}
$$

By induction argument, we reach

$$
\begin{aligned}
V^{*}\left(t_{k}\right) \leq & V^{*}\left(t_{k-1}\right)+(\gamma+\lambda) \int_{t_{k-1}}^{t_{k}} V^{*}(s) d s \\
& +h \rho e^{\gamma \tau} \sum_{j=1}^{n} \int_{t_{k-1}-\tau_{j}\left(t_{k-1}\right)}^{t_{k}-\tau_{j}\left(t_{k}\right)} e^{\gamma\left(s-t_{0}\right)} V_{j}(s) d s,
\end{aligned}
$$

$$
V^{*}\left(t_{2}\right) \leq V^{*}\left(t_{1}\right)+(\gamma+\lambda) \int_{t_{1}}^{t_{2}} V^{*}(s) d s
$$

$$
+h \rho e^{\gamma \tau} \sum_{j=1}^{n} \int_{t_{1}-\tau_{j}\left(t_{1}\right)}^{t_{2}-\tau_{j}\left(t_{2}\right)} e^{\gamma\left(s-t_{0}\right)} V_{j}(s) d s,
$$

$$
V^{*}\left(t_{1}\right) \leq V^{*}\left(t_{0}\right)+(\gamma+\lambda) \int_{t_{0}}^{t_{1}} V^{*}(s) d s
$$

$$
+h \rho e^{\gamma \tau} \sum_{j=1}^{n} \int_{t_{0}-\tau_{j}\left(t_{0}\right)}^{t_{1}-\tau_{j}\left(t_{1}\right)} e^{\gamma\left(s-t_{0}\right)} V_{j}(s) d s .
$$


Thus,

$$
\begin{aligned}
V^{*}(t) \leq & V^{*}\left(t_{0}\right)+(\gamma+\lambda) \int_{t_{0}}^{t} V^{*}(s) d s \\
& +h \rho e^{\gamma \tau} \sum_{j=1}^{n} \int_{t_{0}-\tau_{j}\left(t_{0}\right)}^{t-\tau_{j}(t)} e^{\gamma\left(s-t_{0}\right)} V_{j}(s) d s \\
\leq & V^{*}\left(t_{0}\right)+(\gamma+\lambda) \int_{t_{0}}^{t} V^{*}(s) d s \\
& +h \rho e^{\gamma \tau} \sum_{j=1}^{n} \int_{t_{0}-\tau_{j}\left(t_{0}\right)}^{t} e^{\gamma\left(s-t_{0}\right)} V_{j}(s) d s \\
= & V^{*}\left(t_{0}\right)+\left(\gamma+\lambda+h \rho e^{\gamma \tau}\right) \int_{t_{0}}^{t} V^{*}(s) d s \\
& +h \rho e^{\gamma \tau} \sum_{j=1}^{n} \int_{t_{0}-\tau_{j}\left(t_{0}\right)}^{t_{0}} e^{\gamma\left(s-t_{0}\right)} V_{j}(s) d s, \\
& t \in\left(t_{k}, t_{k+1}\right], k=0,1,2, \ldots
\end{aligned}
$$

Since

$$
\begin{aligned}
& h \rho e^{\gamma \tau} \sum_{j=1}^{n} \int_{t_{0}-\tau_{j}\left(t_{0}\right)}^{t_{0}} e^{\gamma\left(s-t_{0}\right)} V_{j}(s) d s \\
& \quad \leq h \rho e^{\gamma \tau} \sum_{j=1}^{n} \int_{t_{0}-\tau}^{t_{0}} V_{j}(s) d s \\
& \quad=h \rho e^{\gamma \tau} \int_{t_{0}-\tau}^{t_{0}}\left(\sum_{j=1}^{n} \int_{\Omega} \varphi_{j}^{2}(s, x) d x\right) d s \\
& \quad \leq \tau h \rho \mathrm{e}^{\gamma \tau} \overline{\| \varphi}_{\Omega}^{2},
\end{aligned}
$$

we claim

$$
\begin{aligned}
V^{*}(t) \leq & V^{*}\left(t_{0}\right)+\tau h \rho e^{\gamma \tau}{\overline{\|\varphi\|_{\Omega}^{2}}}^{2}+\left(\gamma+\lambda+h \rho e^{\gamma \tau}\right) \int_{t_{0}}^{t} V^{*}(s) d s \\
& \quad t \in\left(t_{k}, t_{k+1}\right], k=0,1,2 \ldots
\end{aligned}
$$

According to Lemma 2, we assert that

$$
\begin{aligned}
V^{*}(t) \leq & \left(V^{*}\left(t_{0}\right)+\tau h \rho e^{\gamma \tau}{\overline{\|\varphi\|_{\Omega}^{2}}}^{2}\right) \\
& \times \exp \left\{\left(\gamma+\lambda+h \rho e^{\gamma \tau}\right)\left(t-t_{0}\right)\right\}, \quad t \geq t_{0},
\end{aligned}
$$

which reduces to

$$
\begin{aligned}
& \left\|u\left(t, x ; t_{0}, \varphi\right)\right\|_{\Omega} \\
& \leq \sqrt{1+\tau h \rho e^{\gamma \tau}} \overline{\|\varphi\|_{\Omega}} \\
& \quad \times \exp \left\{\left(\frac{\lambda+h \rho e^{\gamma \tau}}{2}\right)\left(t-t_{0}\right)\right\}, \quad t \geq t_{0} .
\end{aligned}
$$

This completes the proof.
Remark 6. According to the conditions of Theorem 5, we see that the reaction-diffusion terms can influence the stability of equilibrium point $u=0$. Specifically, the acting factors include the reaction-diffusion coefficients and the first eigenvalue of corresponding Dirichlet Laplacian.

Remark 7. It is not difficult to see that there must exist constant $\gamma>0$ satisfying condition 3 of Theorem 5 if $\lambda<-h \rho$.

Theorem 8. Assume the following.

(1) $\underline{D}=\min \left\{D_{i s}: i=1, \ldots, n ; s=1, \ldots, m\right\}>0$ and denote $2 \underline{D} \lambda_{1}=\chi$.

(2) $P_{i k}\left(u_{i}\left(t_{k}, x\right)\right)=-\theta_{i k} u_{i}\left(t_{k}, x\right), 1-\sqrt{1+\alpha} \leq \theta_{i k} \leq 1+$ $\sqrt{1+\alpha}, \alpha \geq 0$.

(3) $\inf _{k=1,2 \ldots}\left(t_{k}-t_{k-1}\right) \geq \mu$.

(4) There exists a constant $\gamma>0$ satisfying $\gamma+\lambda+h \rho e^{\gamma \tau}>$ 0 and $\lambda+h \rho e^{\gamma \tau}+\ln (1+\alpha) / \mu<0$, where $\lambda=$ $\max _{i=1, \ldots, n}\left(-\chi-2 \underline{a}_{i} p_{i}+\bar{a}_{i} \sum_{j=1}^{n} b_{i j}^{2}+\bar{a}_{i} \sum_{j=1}^{n} c_{i j}^{2}\right)+\rho$ and $\rho=\max _{i=1, \ldots, n}\left(l_{i}^{2}\right) \sum_{i=1}^{n} \bar{a}_{i}$.

Then, the equilibrium point $u=0$ of systems (1)-(2) is globally exponentially stable with convergence rate $-(1 / 2)(\lambda+$ $\left.h \rho \mathrm{e}^{\gamma \tau}+\ln (1+\alpha) / \mu\right)$.

Proof. Define Lyapunov function $V$ of the form $V(t)=$ $\sum_{i=1}^{n} V_{i}(t)$, where $V_{i}(t)=\int_{\Omega} u_{i}^{2}(t, x) d x$. Obviously, $V(t)$ is a piecewise continuous function with the first kind discontinuous points $t_{k}, k=1,2, \ldots$, where it is continuous from the left; that is, $V\left(t_{k}-0\right)=V\left(t_{k}\right)(k=1,2, \ldots)$. Furthermore, for $t=t_{k}(k=0,1,2, \ldots)$, we derive from condition 2 of Theorem 8 that

$$
\begin{aligned}
& u_{i}^{2}\left(t_{k}+0, x\right)-u_{i}^{2}\left(t_{k}, x\right) \\
& \quad=\left(1-\theta_{i k}\right)^{2} u_{i}^{2}\left(t_{k}, x\right)-u_{i}^{2}\left(t_{k}, x\right) \leq \alpha u_{i}^{2}\left(t_{k}, x\right) .
\end{aligned}
$$

Thereby,

$$
V\left(t_{k}+0\right) \leq \alpha V\left(t_{k}\right)+V\left(t_{k}\right), \quad k=0,1,2, \ldots
$$

Construct function $V^{*}(t)=e^{\gamma\left(t-t_{0}\right)} V(t)$ again, where $\gamma>$ 0 satisfies $\gamma+\lambda+h \rho e^{\gamma \tau}>0$ and $\lambda+h \rho e^{\gamma \tau}+\ln (1+\alpha) / \mu<$ 0 . Then, $V^{*}(t)$ is also a piecewise continuous function with the first kind discontinuous points $t_{k}, k=1,2, \ldots$, where it is continuous from the left; that is, $V^{*}\left(t_{k}-0\right)=V^{*}\left(t_{k}\right)(k=$ $1,2, \ldots)$. And for $t=t_{k}(k=0,1,2, \ldots)$, it follows from $(45)$ that

$$
V^{*}\left(t_{k}+0\right) \leq \alpha V^{*}\left(t_{k}\right)+V^{*}\left(t_{k}\right), \quad k=0,1,2, \ldots
$$

Set $t \in\left(t_{k}, t_{k+1}\right], k=0,1,2, \ldots$. Following the same procedure as shown in the proof of Theorem 5 , we get

$$
\begin{aligned}
& V^{*}(t) \leq V^{*}\left(t_{k}+0\right)+(\gamma+\lambda) \int_{t_{k}}^{t} V^{*}(s) d s \\
&+h \rho e^{\gamma \tau} \sum_{j=1}^{n} \int_{t_{k}-\tau_{j}\left(t_{k}\right)}^{t-\tau_{j}(t)} e^{\gamma\left(\theta-t_{0}\right)} V_{j}(\theta) d \theta \\
& t \in\left(t_{k}, t_{k+1}\right], k=0,1,2, \ldots
\end{aligned}
$$


The relations (46) and (47) yield

$$
\begin{aligned}
& V^{*}(t)-V^{*}\left(t_{k}\right) \\
& \leq \alpha V^{*}\left(t_{k}\right)+(\gamma+\lambda) \int_{t_{k}}^{t} V^{*}(s) d s \\
& +h \rho e^{\gamma \tau} \sum_{j=1}^{n} \int_{t_{k}-\tau_{j}\left(t_{k}\right)}^{t-\tau_{j}(t)} e^{\gamma\left(\theta-t_{0}\right)} V_{j}(\theta) d \theta \\
& t \in\left(t_{k}, t_{k+1}\right], k=0,1,2, \ldots
\end{aligned}
$$

By induction argument, we obtain

$$
\begin{aligned}
& V^{*}\left(t_{k}\right)-V^{*}\left(t_{k-1}\right) \\
& \leq \alpha V^{*}\left(t_{k-1}\right)+(\gamma+\lambda) \int_{t_{k-1}}^{t_{k}} V^{*}(s) d s \\
&+h \rho e^{\gamma \tau} \sum_{j=1}^{n} \int_{t_{k-1}-\tau_{j}\left(t_{k-1}\right)}^{t_{k}-\tau_{j}\left(t_{k}\right)} e^{\gamma\left(\theta-t_{0}\right)} V_{j}(\theta) d \theta, \\
& \vdots \\
& V^{*}\left(t_{2}\right)-V^{*}\left(t_{1}\right) \\
& \leq \alpha V^{*}\left(t_{1}\right)+(\gamma+\lambda) \int_{t_{1}}^{t_{2}} V^{*}(s) d s \\
&+h \rho e^{\gamma \tau} \sum_{j=1}^{n} \int_{t_{1}-\tau_{j}\left(t_{1}\right)}^{t_{2}-\tau_{j}\left(t_{2}\right)} e^{\gamma\left(\theta-t_{0}\right)} V_{j}(\theta) d \theta, \\
& V^{*}\left(t_{1}\right)-V^{*}\left(t_{0}\right) \\
& \leq \alpha V^{*}\left(t_{0}\right)+\left(\gamma \rho e^{\gamma \tau} \sum_{j=1}^{n} \int_{t_{0}-\tau_{j}\left(t_{0}\right)}^{t_{1}-\tau_{j}\left(t_{1}\right)} e^{\gamma\left(\theta-t_{0}\right)} V_{j}(\theta) d \theta .\right. \\
& t_{0}
\end{aligned}
$$

Hence,

$$
\begin{aligned}
& V^{*}(t)-V^{*}\left(t_{0}\right) \\
& \leq \alpha V^{*}\left(t_{0}\right)+(\gamma+\lambda) \int_{t_{0}}^{t} V^{*}(s) d s \\
& \quad+h \rho e^{\gamma \tau} \sum_{j=1}^{n} \int_{t_{0}-\tau_{j}\left(t_{0}\right)}^{t-\tau_{j}(t)} e^{\gamma\left(\theta-t_{0}\right)} V_{j}(\theta) d \theta \\
& \quad+\alpha \sum_{t_{0}<t_{k}<t} V\left(t_{k}\right)
\end{aligned}
$$

$$
\begin{gathered}
\leq \alpha V^{*}\left(t_{0}\right)+\left(\gamma+\lambda+h \rho e^{\gamma \tau}\right) \int_{t_{0}}^{t} V^{*}(s) d s \\
+h \rho e^{\gamma \tau} \sum_{j=1}^{n} \int_{t_{0}-\tau_{j}\left(t_{0}\right)}^{t_{0}} e^{\gamma\left(\theta-t_{0}\right)} V_{j}(\theta) d \theta+\alpha \sum_{t_{0}<t_{k}<t} V\left(t_{k}\right), \\
t \in\left(t_{k}, t_{k+1}\right], k=0,1,2, \ldots
\end{gathered}
$$

Introducing $h \rho e^{\gamma \tau} \sum_{j=1}^{n} \int_{t_{0}-\tau_{j}\left(t_{0}\right)}^{t_{0}} e^{\gamma\left(\theta-t_{0}\right)} V_{j}(\theta) d \theta \leq$ $\tau h \rho e^{\gamma \tau} \overline{\| \varphi}_{\Omega}^{2}$ as shown in the proof of Theorem 5 into (50), (50) becomes, for $t \in\left(t_{k}, t_{k+1}\right], k=0,1,2, \ldots$,

$$
\begin{aligned}
V^{*}(t) & -V^{*}\left(t_{0}\right) \\
\leq & \alpha V^{*}\left(t_{0}\right)+\tau h \rho e^{\gamma \tau}\|\varphi\|_{\Omega}^{2} \\
& +\left(\gamma+\lambda+h \rho e^{\gamma \tau}\right) \int_{t_{0}}^{t} V^{*}(s) d s+\alpha \sum_{t_{0}<t_{k}<t} V\left(t_{k}\right) .
\end{aligned}
$$

It then results from Lemma 2 that, for $t \geq t_{0}$,

$$
\begin{aligned}
V^{*}(t) \leq & \left((\alpha+1) V^{*}\left(t_{0}\right)+\tau h \rho e^{\gamma \tau}{\overline{\|\varphi\|_{\Omega}^{2}}}^{2}\right) \\
& \times \prod_{t_{0}<t_{k}<t}(1+\alpha) \exp \left(\left(\gamma+\lambda+h \rho e^{\gamma \tau}\right)\left(t-t_{0}\right)\right) \\
= & \left((\alpha+1) V^{*}\left(t_{0}\right)+\tau h \rho e^{\gamma \tau}{\overline{\|\varphi\|_{\Omega}^{2}}}^{2}\right. \\
& \times(1+\alpha)^{k} \exp \left(\left(\gamma+\lambda+h \rho e^{\gamma \tau}\right)\left(t-t_{0}\right)\right) .
\end{aligned}
$$

On the other hand, since $\inf _{k=1,2, \ldots}\left(t_{k}-t_{k-1}\right) \geq \mu$, one has $k \leq\left(t_{k}-t_{0}\right) / \mu$. Thereby,

$$
\begin{aligned}
(1+\alpha)^{k} & \leq \exp \left\{\frac{\ln (1+\alpha)}{\mu}\left(t_{k}-t_{0}\right)\right\} \\
& \leq \exp \left\{\frac{\ln (1+\alpha)}{\mu}\left(t-t_{0}\right)\right\}
\end{aligned}
$$

and (52) can be rewritten as

$$
\begin{aligned}
V^{*}(t) \leq & \left((\alpha+1) V^{*}\left(t_{0}\right)+\tau h \rho e^{\gamma \tau}{\overline{\|\varphi\|_{\Omega}^{2}}}^{2}\right. \\
& \times \exp \left(\left(\gamma+\lambda+h \rho e^{\gamma \tau}+\frac{\ln (1+\alpha)}{\mu}\right)\left(t-t_{0}\right)\right)
\end{aligned}
$$

which implies

$$
\begin{aligned}
& \left\|u\left(t, x ; t_{0}, \varphi\right)\right\|_{\Omega} \leq \sqrt{\left(\alpha+1+\tau h \rho e^{\gamma \tau}\right)} \overline{\|\varphi\|_{\Omega}} \\
& \quad \times \exp \left(\frac{1}{2}\left(\lambda+h \rho e^{\gamma \tau}+\frac{\ln (1+\alpha)}{\mu}\right)\left(t-t_{0}\right)\right), \quad t \geq t_{0} .
\end{aligned}
$$

The proof is completed. 
Due to Lemma 4, we know that the following inequalities:

$$
\begin{aligned}
& 2 \sum_{j=1}^{n} b_{i j} \int_{\Omega} u_{i}(t, x) f\left(u_{j}(t, x)\right) d x \\
& \quad \leq \sum_{j=1}^{n} \int_{\Omega}\left(\varepsilon_{1} b_{i j}^{2} u_{i}^{2}(t, x)+\frac{l_{j}^{2}}{\varepsilon_{1}} u_{j}^{2}(t, x)\right) d x, \\
& 2 \sum_{j=1}^{n} c_{i j} \int_{\Omega} u_{i}(t, x) f\left(u_{j}\left(t-\tau_{j}, x\right)\right) d x \\
& \quad \leq \sum_{j=1}^{n} \int_{\Omega}\left(\varepsilon_{2} c_{i j}^{2} u_{i}^{2}(t, x)+\frac{l_{j}^{2}}{\varepsilon_{2}} u_{i}^{2}\left(t-\tau_{j}, x\right)\right) d x
\end{aligned}
$$

hold for any $\varepsilon_{1}, \varepsilon_{2}>0$. Thus, in a similar way to the proofs of Theorems 5-8, we can prove the following theorems.

Theorem 9. Assume the following.

(1) $\underline{D}=\min \left\{D_{i s}: i=1, \ldots, n ; s=1, \ldots, m\right\}>0$ and denote $2 \underline{D} \lambda_{1}=\chi$.

(2) $P_{i k}\left(u_{i}\left(t_{k}, x\right)\right)=-\theta_{i k} u_{i}\left(t_{k}, x\right), 0 \leq \theta_{i k} \leq 2$.

(3) There exist constants $\gamma>0$ and $\varepsilon_{1}, \varepsilon_{2}>0$ such that $\gamma+\lambda+h \rho e^{\gamma \tau}>0$ and $\lambda+h \rho e^{\gamma \tau}<0$, where $\lambda=$ $\max _{i=1, \ldots, n}\left(-\chi-2 \underline{a}_{i} p_{i}+\bar{a}_{i} \sum_{j=1}^{n}\left(\varepsilon_{1} b_{i j}^{2}+\varepsilon_{2} c_{i j}^{2}\right)\right)+$ $\left(\max _{i=1, \ldots, n}\left(l_{i}^{2}\right) / \varepsilon_{1}\right) \sum_{i=1}^{n} \bar{a}_{i}$, and $\rho=\left(\max _{i=1, \ldots, n}\left(l_{j}^{2}\right) / \varepsilon_{2}\right)$ $\sum_{i=1}^{n} \bar{a}_{i}$

Then, the equilibrium point $u=0$ of systems (1)-(2) is globally exponentially stable with convergence rate $-\left(\lambda+h \rho e^{\gamma \tau}\right) / 2$.

Remark 10. There must exist constant $\gamma>0$ satisfying condition 3 of Theorem 9 if there are constants $\varepsilon_{1}, \varepsilon_{2}>0$ such that $\lambda<-h \rho$.

Theorem 11. Assume the following.

(1) $\underline{D}=\min \left\{D_{i s}: i=1, \ldots, n ; s=1, \ldots, m\right\}>0$ and denote $2 \underline{D} \lambda_{1}=\chi$.

(2) $P_{i k}\left(u_{i}\left(t_{k}, x\right)\right)=-\theta_{i k} u_{i}\left(t_{k}, x\right), 1-\sqrt{1+\alpha} \leq \theta_{i k} \leq 1+$ $\sqrt{1+\alpha}, \alpha \geq 0$.

(3) $\inf _{k=1,2, \ldots}\left(t_{k}-t_{k-1}\right) \geq \mu$.

(4) There exist constants $\gamma>0$ and $\varepsilon_{1}, \varepsilon_{2}>0$ satisfying $\gamma+\lambda+h \rho e^{\gamma \tau}>0$ and $\lambda+h \rho e^{\gamma \tau}+\ln (1+\alpha) / \mu<0$, where

$$
\begin{gathered}
\lambda=\max _{i=1, \ldots, n}\left(-\chi-2 \underline{a}_{i} p_{i}+\bar{a}_{i} \sum_{j=1}^{n}\left(\varepsilon_{1} b_{i j}^{2}+\varepsilon_{2} c_{i j}^{2}\right)\right) \\
+\frac{\max _{i=1, \ldots, n}\left(l_{i}^{2}\right)}{\varepsilon_{1}} \sum_{i=1}^{n} \bar{a}_{i}, \\
\rho=\frac{\max _{i=1, \ldots, n}\left(l_{i}^{2}\right)}{\varepsilon_{2}} \sum_{i=1}^{n} \bar{a}_{i} .
\end{gathered}
$$

Then, the equilibrium point $u=0$ of systems (1)-(2) is globally exponentially stable with convergence rate $-(1 / 2)\left(\lambda+h \rho e^{\gamma \tau}+\right.$ $\ln (1+\alpha) / \mu)$.

Further, on the condition that $\left|P_{i k}\left(u_{i}\left(t_{k}, x\right)\right)\right| \leq \theta_{i k}\left|u_{i}\left(t_{k}, x\right)\right|$, where $\theta_{i k}^{2}<(\alpha-1) / 2$ and $\alpha \geq 1$, we obtain

$$
\begin{aligned}
u_{i}^{2}\left(t_{k}\right. & +0, x)-u_{i}^{2}\left(t_{k}, x\right) \\
& \leq 2\left(u_{i}\left(t_{k}, x\right)\right)^{2}+2\left(P_{i k}\left(u_{i}\left(t_{k}, x\right)\right)\right)^{2}-u_{i}^{2}\left(t_{k}, x\right) \\
& \leq\left(2+2 \theta_{i k}^{2}\right)\left(u_{i}\left(t_{k}, x\right)\right)^{2}-u_{i}^{2}\left(t_{k}, x\right) \leq \alpha u_{i}^{2}\left(t_{k}, x\right)
\end{aligned}
$$

for $t=t_{k}(k=1,2, \ldots)$. In an identical way with the proof of Theorem 8, we can present the following.

Theorem 12. Assume the following.

(1) Let $\underline{D}=\min \left\{D_{i s}: i=1, \ldots, n ; s=1, \ldots, m\right\}>0$ and denote $2 \underline{D} \lambda_{1}=\chi$.

(2) $\left|P_{i k}\left(u_{i}\left(t_{k}, x\right)\right)\right| \leq \theta_{i k}\left|u_{i}\left(t_{k}, x\right)\right|$, where $\theta_{i k}^{2} \leq(\alpha-1) / 2$ and $\alpha \geq 1$.

(3) $\inf _{k=1,2, \ldots}\left(t_{k}-t_{k-1}\right) \geq \mu$.

(4) There exist constants $\gamma>0$ and $\varepsilon_{1}, \varepsilon_{2}>0$ such that $\gamma+\lambda+h \rho e^{\gamma \tau}>0$ and $\lambda+h \rho e^{\gamma \tau}+\ln (1+\alpha) / \mu<0$, where

$$
\begin{gathered}
\lambda=\max _{i=1, \ldots, n}\left(-\chi-2 \underline{a}_{i} p_{i}+\bar{a}_{i} \sum_{j=1}^{n}\left(\varepsilon_{1} b_{i j}^{2}+\varepsilon_{2} c_{i j}^{2}\right)\right) \\
+\frac{\max _{i=1, \ldots, n}\left(l_{i}^{2}\right)}{\varepsilon_{1}} \sum_{i=1}^{n} \bar{a}_{i}, \\
\rho=\frac{\max _{i=1, \ldots, n}\left(l_{i}^{2}\right)}{\varepsilon_{2}} \sum_{i=1}^{n} \bar{a}_{i} .
\end{gathered}
$$

Then, the equilibrium point $u=0$ of systems (1)-(2) is globally exponentially stable with convergence rate $-(1 / 2)\left(\lambda+h \rho e^{\gamma \tau}+\right.$ $\ln (1+\alpha) / \mu)$.

Remark 13. Different from Theorems 5-11, the impulsive part in Theorem 12 could be nonlinear, and this will be of more applicability. Actually, Theorems 5-11 can be regarded as the special cases of Theorem 12 .

\section{Examples}

Example 14. Consider problems (1)-(4) with $P_{i k}\left(u_{i}\left(t_{k}, x\right)\right)=$ 1.343u $u_{i}\left(t_{k}, x\right)$; moreover, $n=2, m=2, \Omega=\left\{\left(x_{1}, x_{2}\right)^{T}\right.$ | $\left.x_{1}^{2}+x_{2}^{2}<1\right\}, a_{i}\left(u_{1}(t, x)\right)=1, \omega_{1}\left(u_{1}(t, x)\right)=6.5 u_{1}(t, x)$, $\omega_{2}\left(u_{2}(t, x)\right)=8.5 u_{2}(t, x),\left(D_{i s}\right)=\left(\begin{array}{ll}1.2 & 2.3 \\ 2.2 & 1.5\end{array}\right),\left(b_{i j}\right)=\left(\begin{array}{ll}-0.23 & 1.3 \\ -0.14 & 3.2\end{array}\right)$, $\left(c_{i j}\right)=\left(\begin{array}{cc}-0.1 & -0.2 \\ 0.25 & -0.13\end{array}\right), f_{j}\left(u_{j}\right)=(\sqrt{2} / 4)\left(\left|u_{j}+1\right|-\left|u_{j}-1\right|\right)$, and $\tau_{j}(t)=(3 / 4) \arctan (t)$. 
As $\lambda_{1}=5.783$ and $\underline{D}=1.2$, we know $\chi=13.8792$. Further, for $l_{i}=\sqrt{2} / 2, \underline{a}_{i}=\overline{a_{i}}=1, p_{1}=6.5$, and $p_{2}=8.5$, we compute

$$
\begin{gathered}
\rho=\max _{i=1, \ldots, n}\left(l_{i}^{2}\right) \sum_{i=1}^{n} \bar{a}_{i}=1, \\
\lambda=\max _{i=1, \ldots, n}\left(-\chi-2 \underline{a}_{i} p_{i}+\bar{a}_{i} \sum_{j=1}^{n} b_{i j}^{2}+\bar{a}_{i} \sum_{j=1}^{n} c_{i j}^{2}\right) \\
+\rho=-15.5402 .
\end{gathered}
$$

Let $h=4$. Since $\lambda=-15.5402<-4=-h \rho$, we therefore conclude from Theorem 5 that the zero solution of this system is globally exponential stable.

Example 15. Consider problems (1)-(4) with $P_{i k}\left(u_{i}\left(t_{k}, x\right)\right)=$ $\arctan \left(0.5 u_{i}\left(t_{k}, x\right)\right)$; moreover, $n=2, m=2, \Omega=$ $\left\{\left(x_{1}, x_{2}\right)^{T} \mid x_{1}^{2}+x_{1}^{2}<1\right\}, a_{i}\left(u_{1}(t, x)\right)=1, \omega_{1}\left(u_{1}(t, x)\right)=$ $6.5 u_{1}(t, x), \omega_{2}\left(u_{2}(t, x)\right)=8.5 u_{2}(t, x),\left(D_{i s}\right)=\left(\begin{array}{ll}1.2 & 2.3 \\ 2.2 & 1.5\end{array}\right),\left(b_{i j}\right)=$ $\left(\begin{array}{ll}-0.23 & 1.3 \\ -0.14 & 3.2\end{array}\right),\left(c_{i j}\right)=\left(\begin{array}{cc}-0.1 & -0.2 \\ 0.25 & -0.13\end{array}\right), f_{j}\left(u_{j}\right)=(\sqrt{2} / 4)\left(\left|u_{j}+1\right|-\mid u_{j}-\right.$ $1 \mid), \tau_{j}(t)=(1 / \pi) \arctan (t)$, and $t_{k}=t_{k-1}+k$.

As $\lambda_{1}=5.783$ and $\underline{D}=1.2$, we know $\chi=13.8792$. Further, for $l_{i}=\sqrt{2} / 2, \underline{a}_{i}=\overline{a_{i}}=1, p_{1}=6.5, p_{2}=8.5$, and $\varepsilon_{i}=1$, we compute

$$
\begin{gathered}
\rho=\frac{\max _{i=1, \ldots, n}}{\varepsilon_{2}} \sum_{i=1}^{n} \bar{a}_{i}=1, \\
\lambda=\max _{i=1, \ldots, n}\left(-\chi-2 \underline{a}_{i} p_{i}+\bar{a}_{i} \sum_{j=1}^{n}\left(\varepsilon_{1} b_{i j}^{2}+\varepsilon_{2} c_{i j}^{2}\right)\right) \\
+\frac{\max _{i=1, \ldots, n}\left(l_{i}^{2}\right)}{\varepsilon_{1}} \sum_{i=1}^{n} \bar{a}_{i}=-15.5402 .
\end{gathered}
$$

Let $\tau=0.5, h=4, \mu=1, \theta_{i k}=0.5$, and $\alpha=1.5$; we can find $\gamma=2.4$ such that

$$
\begin{aligned}
& \gamma+\lambda+h \rho e^{\gamma \tau}=0.1403>0, \\
& \lambda+h \rho e^{\gamma \tau}+\frac{\ln (1+\alpha)}{\mu}=-1.3434<0 .
\end{aligned}
$$

Therefore it is concluded from Theorem 12 that the zero solution of this system is globally exponential stable.

\section{References}

[1] M. A. Cohen and S. Grossberg, "Absolute stability of global pattern formation and parallel memory storage by competitive neural networks," IEEE Transactions on Systems, Man, and Cybernetics, vol. 13, no. 5, pp. 815-826, 1983.

[2] J. Cao and J. Liang, "Boundedness and stability for CohenGrossberg neural network with time-varying delays," Journal of Mathematical Analysis and Applications, vol. 296, no. 2, pp. 665685, 2004.

[3] J. Cao and J. Wang, "Global exponential stability and periodicity of recurrent neural networks with time delays," IEEE Transactions on Circuits and Systems. I., vol. 52, no. 5, pp. 920-931, 2005.
[4] X. Liu, "Stability results for impulsive differential systems with applications to population growth models," Dynamics and Stability of Systems, vol. 9, no. 2, pp. 163-174, 1994.

[5] X. Liu and Q. Wang, "Impulsive stabilization of high-order Hopfield-type neural networks with time-varying delays," IEEE Transactions on Neural Networks, vol. 19, no. 1, pp. 71-79, 2008.

[6] Z. Yang and D. Xu, "Impulsive effects on stability of CohenGrossberg neural networks with variable delays," Applied Mathematics and Computation, vol. 177, no. 1, pp. 63-78, 2006.

[7] S. Arik and Z. Orman, "Global stability analysis of CohenGrossberg neural networks with time varying delays," Physics Letters A, vol. 341, no. 5-6, pp. 410-421, 2005.

[8] T. Huang, C. Li, and G. Chen, "Stability of Cohen-Grossberg neural networks with unbounded distributed delays," Chaos, Solitons \& Fractals, vol. 34, no. 3, pp. 992-996, 2007.

[9] X. Liao, C. Li, and K. W. Wong, "Criteria for exponential stability of Cohen-Grossberg neural networks," Neural Networks, vol. 17, no. 10, pp. 1401-1414, 2004.

[10] J. Zhang, Y. Suda, and H. Komine, "Global exponential stability of Cohen-Grossberg neural networks with variable delays," Physics Letters A, vol. 338, no. 1, pp. 44-50, 2005.

[11] W. Zhang, Y. Tang, J. A. Fang, and X. Wu, "Stability of delayed neural networks with time-varying impulses," Neural Networks, vol. 36, pp. 59-63, 2012.

[12] X. Lai and Y. Zhang, "Fixed point and asymptotic analysis of cellular neural networks," Journal of Applied Mathematics, vol. 2012, Article ID 689845, 12 pages, 2012.

[13] Z. Chen and J. Ruan, "Global stability analysis of impulsive Cohen' Grossberg neural networks with delay," Physics Letters A, vol. 345, pp. 101-111, 2005.

[14] Z. Chen and J. Ruan, "Global dynamic analysis of general Cohen-Grossberg neural networks with impulse," Chaos, Solitons and Fractals, vol. 32, no. 5, pp. 1830-1837, 2007.

[15] Z. Yang and D. Xu, "Impulsive effects on stability of CohenGrossberg neural networks with variable delays," Applied Mathematics and Computation, vol. 177, no. 1, pp. 63-78, 2006.

[16] Q. Song and J. Zhang, "Global exponential stability of impulsive Cohen-Grossberg neural network with time-varying delays," Nonlinear Analysis: Real World Applications, vol. 9, no. 2, pp. 500-510, 2008.

[17] Z. Chen and J. Ruan, "Global dynamic analysis of general Cohen-Grossberg neural networks with impulse," Chaos, Solitons and Fractals, vol. 32, no. 5, pp. 1830-1837, 2007.

[18] Y. Zhang and Q. Luo, "Global exponential stability of impulsive cellular neural networks with time-varying delays via fixed point theory," Advances in Difference Equations, vol. 2013, article 23, 2013.

[19] X. Zhang, S. Wu, and K. Li, "Delay-dependent exponential stability for impulsive Cohen-Grossberg neural networks with time-varying delays and reaction-diffusion terms," Communications in Nonlinear Science and Numerical Simulation, vol. 16, no. 3, pp. 1524-1532, 2011.

[20] J. Pan and S. Zhong, "Dynamical behaviors of impulsive reaction-diffusion Cohen-Grossberg neural network with delays," Neurocomputing, vol. 73, no. 7-9, pp. 1344-1351, 2010.

[21] K. Li and Q. Song, "Exponential stability of impulsive CohenGrossberg neural networks with time-varying delays and reaction-diffusion terms," Neurocomputing, vol. 72, no. 1-3, pp. 231-240, 2008.

[22] J. Qiu, "Exponential stability of impulsive neural networks with time-varying delays and reaction-diffusion terms," Neurocomputing, vol. 70, no. 4-6, pp. 1102-1108, 2007. 
[23] X. Wang and D. Xu, "Global exponential stability of impulsive fuzzy cellular neural networks with mixed delays and reactiondiffusion terms," Chaos, Solitons \& Fractals, vol. 42, no. 5, pp. 2713-2721, 2009.

[24] W. Zhu, "Global exponential stability of impulsive reactiondiffusion equation with variable delays," Applied Mathematics and Computation, vol. 205, no. 1, pp. 362-369, 2008.

[25] Z. Li and K. Li, "Stability analysis of impulsive Cohen-Grossberg neural networks with distributed delays and reaction-diffusion terms," Applied Mathematical Modelling, vol. 33, no. 3, pp. 13371348, 2009.

[26] Z. Li and K. Li, "Stability analysis of impulsive fuzzy cellular neural networks with distributed delays and reaction-diffusion terms," Chaos, Solitons and Fractals, vol. 42, no. 1, pp. 492-499, 2009.

[27] J. Pan, X. Liu, and S. Zhong, "Stability criteria for impulsive reaction-diffusion Cohen-Grossberg neural networks with time-varying delays," Mathematical and Computer Modelling, vol. 51, no. 9-10, pp. 1037-1050, 2010.

[28] Y. Zhang and Q. Luo, "Novel stability criteria for impulsive delayed reaction-diffusion Cohen-Grossberg neural networks via Hardy-Poincarè inequality," Chaos, Solitons \& Fractals, vol. 45, no. 8, pp. 1033-1040, 2012.

[29] Z. Yutian and Z. Minhui, "Stability analysis for impulsive reaction-diffusion Cohen-Grossberg neural networks with time-varying delays," Journal of Nanjing University of Information Science and Technology, vol. 4, no. 3, pp. 213-219, 2012.

[30] V. Lakshmikantham, D. D. Bainnov, and P. S. Simeonov, Theory of Impulsive Differential Equations, vol. 6 of Series in Modern Applied Mathematics, World Scientific Publishing, Singapore, 1989.

[31] D. S. Mitrinovic, Analytic Inequalities, Springer, New York, NY, USA, 1970. 


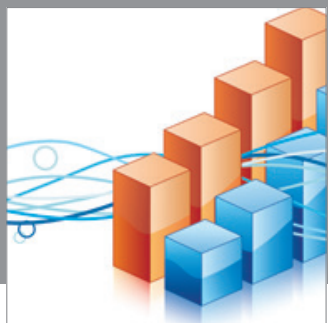

Advances in

Operations Research

mansans

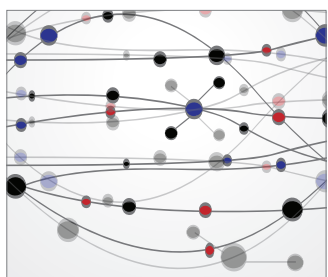

The Scientific World Journal
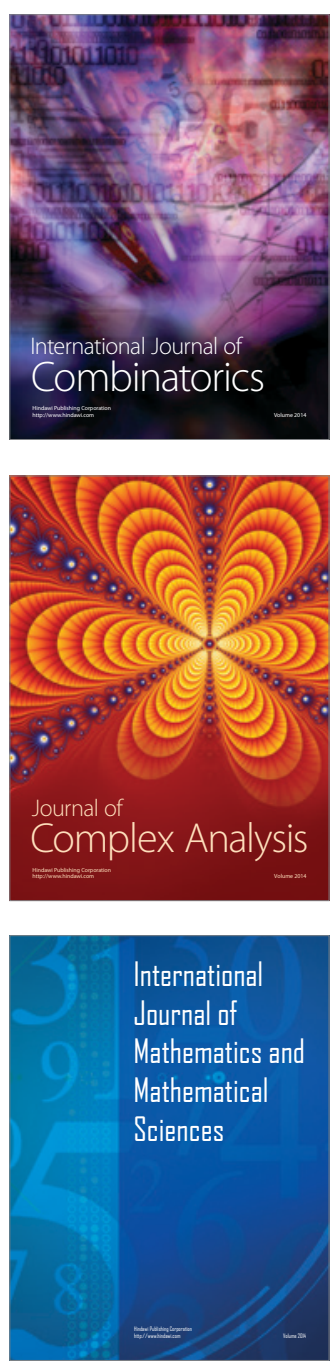
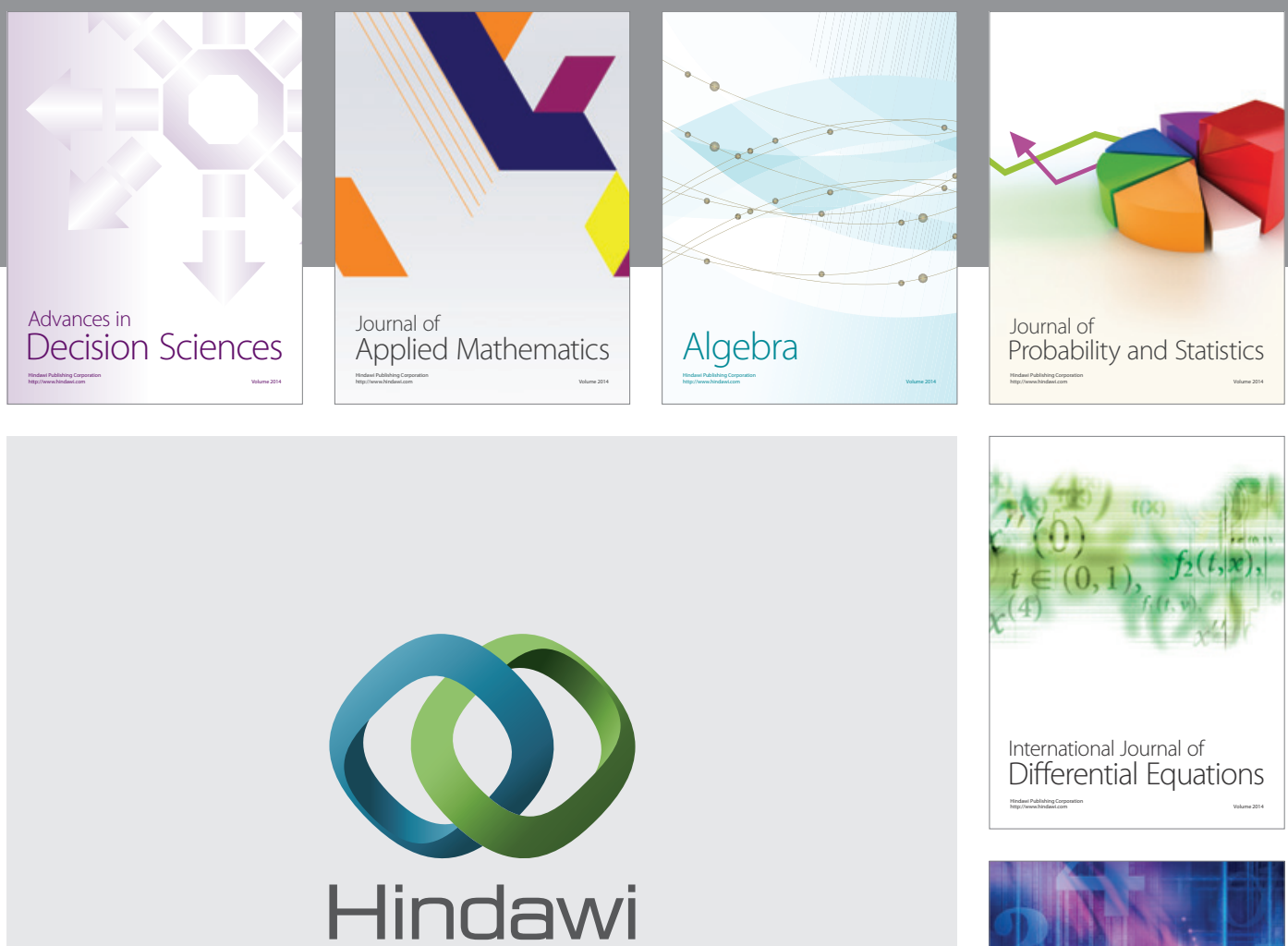

Submit your manuscripts at http://www.hindawi.com
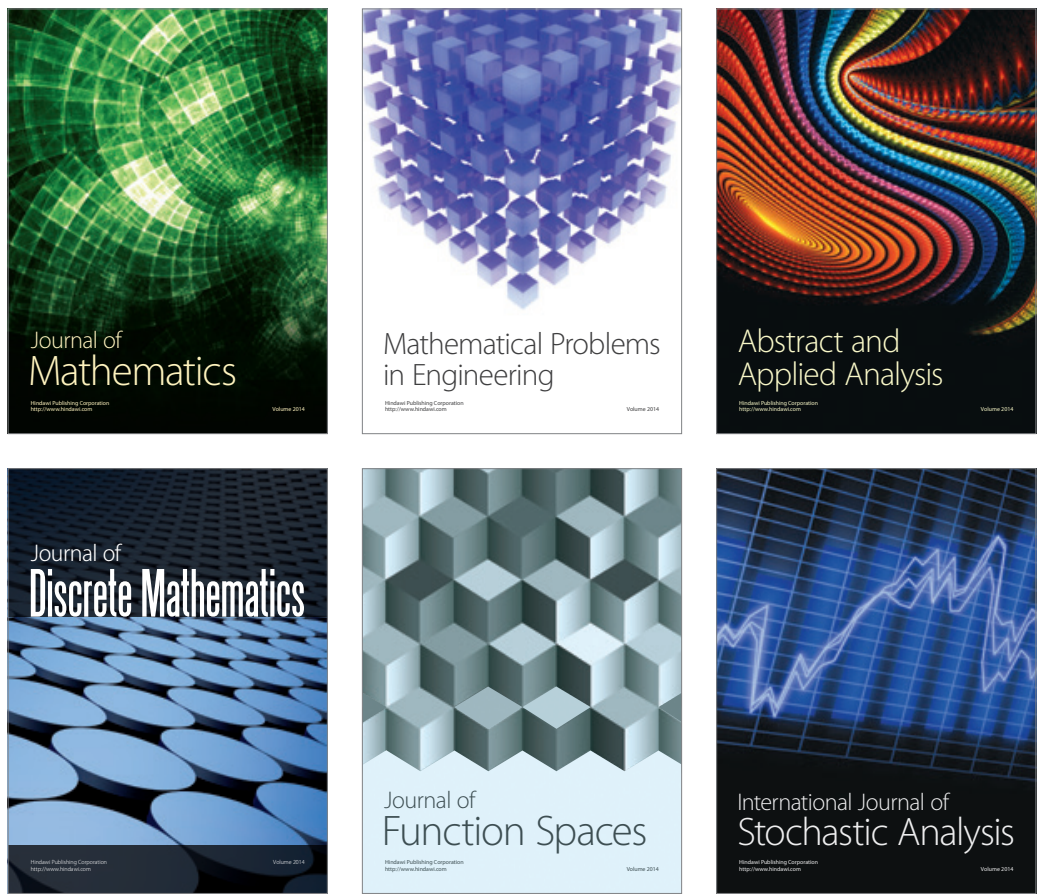

Journal of

Function Spaces

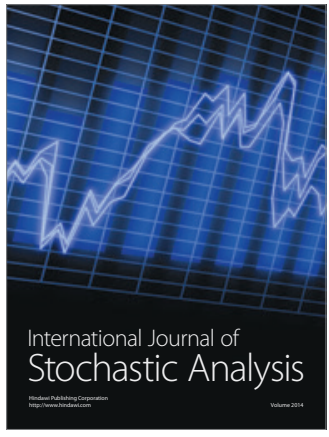

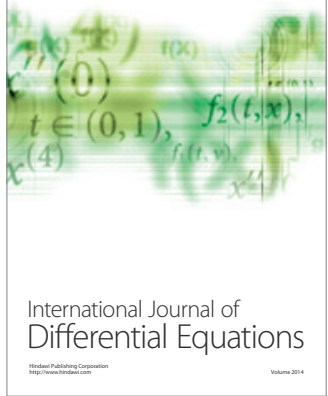
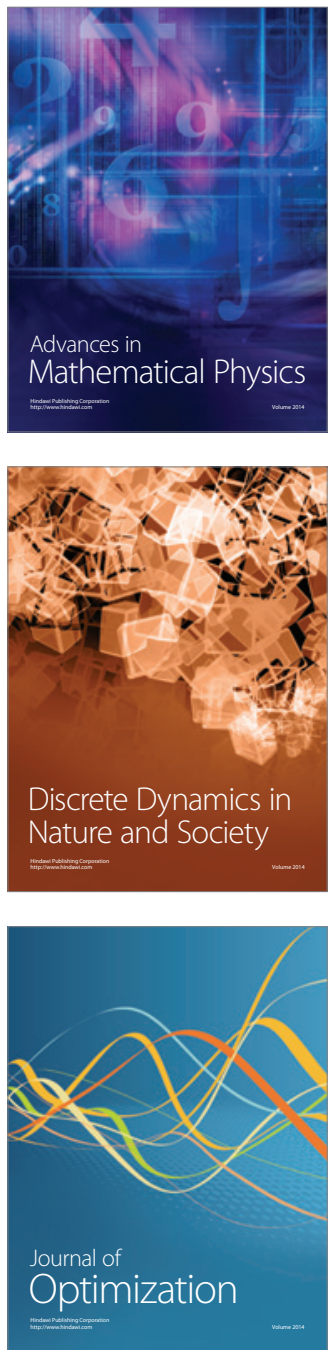